\title{
Modeling of a New Super-Gain BJT and an innovative Low-loss AC Switch based on Gummel-Poon model
}

\author{
Z. Ren, P. Wang, A. Schellmanns, N. Batut and G. Goubard \\ University of Tours, GREMAN UMR-CNRS 7347, France \\ 7 avenue Marcel Dassault, 37200 Tours (France) \\ Phone number: +33 6041470 74, e-mail: zheng.ren@univ-tours.fr
}

\begin{abstract}
This article gives a modeling method based on the Gummel-Poon model for the Super-Gain BJT developed in the GREMAN laboratory. First of all, the extraction principle, mathematical calculating method, and final extraction results of each model parameter will be presented. Secondly, the Gummel and Voltage-Current output curves for a single Super-Gain BJT, which are carried on in Pspice with the extracted parameters in the first part, will be drawn and be compared with experimentation results. At last, a new $600 \mathrm{~V}$ AC switch that is composed of two single Super-Gain BJTs connected in antiseries is simulated. To explain and reduce the error between the simulated results and experimental ones in hi-level injection, a new model with a dynamic resistance based on that of GummelPoon is proposed and validated. Consequently, all of simulations have an error less than $10 \%$, proving the good precision of the models as well as the characteristics of the new AC power switch.
\end{abstract}

\section{Key words}

600V AC Switch, electrical modeling, Gummel-Poon model, Super-Gain BJT, SPICE simulation.

\section{Introduction}

In power electronics, there are already several types of solutions for AC switch. For example, the Triac, IGBT+diodes and MOSFET+diodes are the common methods used in industry. However, their disadvantages are also obvious. According to the report of GREMAN laboratory [1], three main disadvantages are observed in real applications. The half-controllable problem is found in the Triac, the energy dissipation problem in permanent regime influences the performance of the solution IGBT+diodes and for the latest one, MOSFET+diode [2], which makes the cost of the solution expensive.

In this context, the GREMAN laboratory has proposed a new AC switch named 'TBBS' (Transistor Based Bidirectional Switch) which is based on a Super-Gain (forward amplification gain $\mathrm{h}_{\mathrm{FE}}>220$ ) Gate Associated Bipolar Junction Transistor. This new AC switch is possible to be controlled in both directions and its conduction power dissipation is as low as $0.29 \mathrm{~W} / \mathrm{A}$ compared with others having a magnitude around 1-2W/A [3]. In order to confirm the promising characteristics, electrical simulations have been achieved with the simulator Pspice. The single Super-Gain BJT and its combined application 'TBBS' are simulated respectively to verify the modeling and test its robustness. The physical structure of the Super-Gain BJT along with equivalent inner diodes and Gummel-Poon model elements are presented in Fig. 1

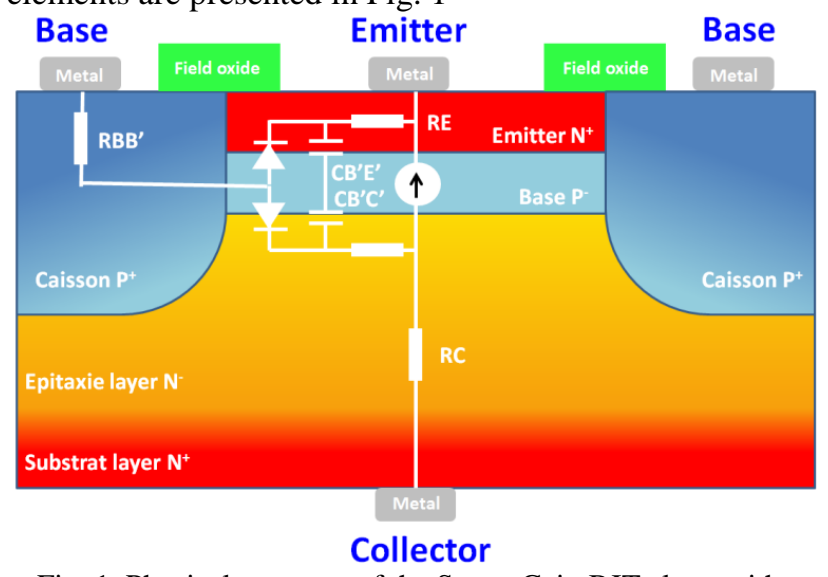

Fig. 1. Physical structure of the Super-Gain BJT along with Gummel-Poon model elements

The TBBS is composed of two Super-Gain BJTs connected in anti-serial, its representative schematic as well as the prototype component is presented as Fig. 2.

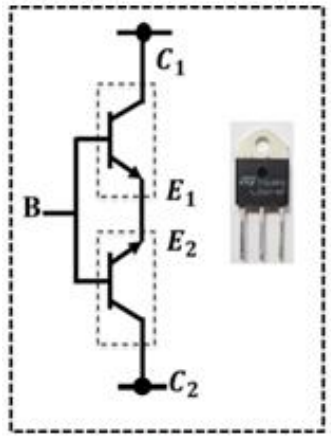

(a)

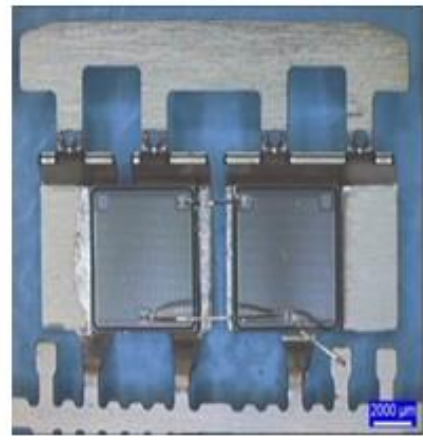

(b)
Fig. 2. Representative schematic of the TBBS (a) and the real prototype component (b)

\section{The super-gain bjt modeling and the presentation of Gummel-Poon model}

As is known, Gummel-poon model was proposed by Hermann Gummel and H. C. Poon in 1970s to simulate 
the working principal of bipolar transistor. The model based on the transistor's physical structure is widely used in SPICE simulation software. This article will focus on the extraction of the resistive, capacitive and DC characteristics (forward bias and reverse bias) parameters of the Gummel-poon model for a Super-Gain BJT.

Before dwelling into the modeling process, some basic mathematical formulas (especially that of Base current $\mathrm{iB}$ and of Collector current $\mathrm{iC}$ ) will be given in the following paragraph to help understand how BJT works. The equivalent circuit for a bipolar transistor is presented in Fig.3. In the model, the diodes and the capacitors replace respectively the PN junctions between different layers as well the capacity between terminals. The parasitic resistances, which locate at the end of terminals, are put into operation at high current.

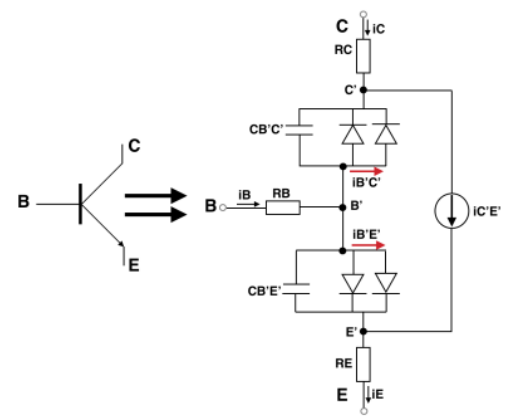

Fig. 3. Gummel-Poon model equivalent electronic circuit for a bipolar transistor

The expression of the base current $\mathrm{iB}$, which is consisted of 4 independent current parts, is given as follow:

$$
\begin{aligned}
i_{B}=\frac{I s}{B F}\left\{\exp \left[\frac{v B E}{N F * v t}\right]-1\right\}+I s e\left\{\exp \left[\frac{v B E}{N E * v t}\right]-1\right\} \\
+\frac{I s}{B R}\left\{\exp \left[\frac{v B C}{N R * v t}\right]-1\right\}+I s c\left\{\exp \left[\frac{v B C}{N c * v t}\right]-1\right\}
\end{aligned}
$$

Similarly, the expression of the collector current iC:

$$
\begin{aligned}
i_{C}= & \frac{I s}{N q B} *\left\{\left[\exp \left(\frac{v B E}{N F * v t}\right)-1\right]-\left[\exp \left(\frac{v B C}{N R * v t}\right)-1\right]\right\} \\
& -\frac{I s}{B R}\left\{\left[\exp \left(\frac{v B C}{N R * v t}\right)-1\right]\right\}-I s c\left\{\exp \left[\frac{v B C}{N c * v t}\right]-1\right\}
\end{aligned}
$$

\section{The parameters extraction of Gummel- Poon model}

In this part, the parameters extraction method will be introduced. The extraction process of each type of parameters will be described in detail. In the case of inverse bias, only the differences relative to direct bias will be introduced to avoid repeating the process.

\section{A. Extraction of the resistive parameters}

There are three ohmic parameters $\left(R_{B}, R_{C}\right.$ and $\left.R_{E}\right)$ in Gummel-Poon model. They represent respectively the resistance of each terminal. The extraction of $\mathrm{R}_{\mathrm{E}}$ uses vCE$i B$ curve (and $v E C-i B$ for $R_{C}$ extraction), here $v C E$ presents the voltage between the electrodes $\mathrm{C}$ and $\mathrm{E}$. Its measuring circuit is shown in Fig.4 (keeping $\mathrm{iC}$ equals to $0 \mathrm{~A}$, varying the input current $\mathrm{iB}$ and reading the voltage vCE through voltmeter).

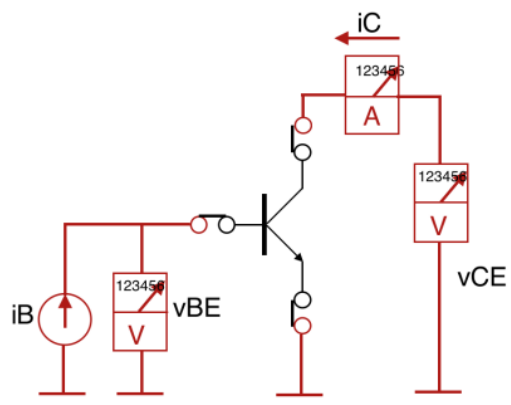

Fig.4 measuring circuit of the resistance Re (when measuring vEC-iB, exchange $\mathrm{CE}$ probes)

The Re, Rc represent respectively the emitter and collector access resistances of a transistor. When there is a current flowing through $\mathrm{PN}$ junction $\mathrm{BE}$ or $\mathrm{BC}$ and finally going back to the source, a voltage drop can be detected in another open-loop terminal. Its amplitude is proportional to the base current $\mathrm{iB}$, which is determined by external drive circuit, and its formula is given as follow:

$$
v C E=V T * \ln (1 / A I)+i_{B} * R_{E}
$$

Here AI is the inverse current amplification in common base mode. According to the formula above, to obtain the value of $\mathrm{Re}$, a simple derivation of variable iB will be performed in both sides of the equation. For each measured data point of $\mathrm{vCE}-\mathrm{iB}$ curve, the derivation result is plotted in the following figure to pick out the value of $\mathrm{Re}$ :

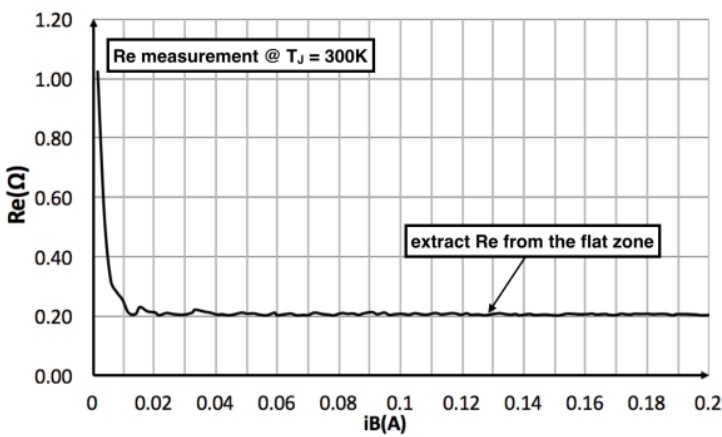

Fig.5 derivation curve of the voltage vCE versus base current iB

For the ohmic parameters extraction, as the derivation (equivalent to $\mathrm{Re}$ ) is a variable of current $\mathrm{iB}$, its final value should be extracted in the flat zone of the curve as shown in Fig.5. In order to improve the accuracy of parameter extraction and to make it more representative and repeatable, numerous transistors are measured and their ohmic parameters are extracted respectively to calculate finally the average value. The extraction result is given in the following table:

Tab-1: extraction results of resistance $\mathrm{Re}$ and $\mathrm{Rc}$

\begin{tabular}{|l|c|c|c|c|c|c|}
\hline & $\begin{array}{l}\text { Chip- } \\
1\end{array}$ & $\begin{array}{l}\text { Chip- } \\
2\end{array}$ & $\begin{array}{l}\text { Chip- } \\
3\end{array}$ & $\begin{array}{l}\text { Chip- } \\
4\end{array}$ & $\begin{array}{l}\text { Chip- } \\
5\end{array}$ & Average \\
\hline $\operatorname{Re}(\Omega)$ & 0.20 & 0.19 & 0.22 & 0.2 & 0.19 & 0.20 \\
\hline $\operatorname{Rc}(\Omega)$ & 0.34 & 0.35 & 0.36 & 0.37 & 0.33 & 0.35 \\
\hline
\end{tabular}

\section{B. Extraction of the capacitive parameters}


The capacitive parameters used to model the PN junction's capacity of accommodating space charge. The model modeling the capacitances of $\mathrm{BE}$ and $\mathrm{BC}$ junctions will be introduced in this paragraph.

The extraction of the capacitive parameters requires the use of an instrument called CV meter. This instrument is capable of measuring the ratio of capacitance $(C)$ and voltage $(\mathrm{V})$ between two BJT terminals, all the capacitive parameters extractions are based on these measured data. It will be connected between two chosen electrodes. For example, connecting $\mathrm{B}$ and $\mathrm{E}$ for Cbe-vBE measurement, and to the opposite, the Cbc-vBE curve is sufficient to connect the CV meter between terminals BC and to keep the rest terminal E 'open'. Combining with the theoretical analysis and the experimentations, the following formulas are used to describe the operation mechanism of space charge between different layers of the super-gain BJT:

For $\mathrm{vBE}<\mathrm{F}_{\mathrm{C}} * \mathrm{~V}_{\mathrm{JE}}$ :

$$
C_{S B E}=\frac{C_{J E}}{\left(1-\frac{v B E}{V_{J E}}\right)^{M_{J E}}}
$$

For other cases:

$$
C_{S B E}=\frac{C_{J E}}{\left(1-F_{C}\right)^{\left(1+M_{J E}\right)}} *\left[1-F_{C} *\left(1+M_{J E}\right)+M_{J E} * \frac{v B E}{V_{J E}}\right]
$$

The working mechanism of the space charges can be divided into two parts (as shown in Eq.4 and Eq.5), and each of them is determined by the applied voltage. Through Eq.4, the parameter $C_{J E}$ can be easily obtained by fixing $\mathrm{vBE}=0 \mathrm{~V}$. The extraction regarding the $\mathrm{Vje}$ and $\mathrm{Mje}$ relies on Eq.5. After the analysis of this equation and measured curve, the method of linear fitting and parameter identification will be applied to determine the value of these two parameters. From Eq.5, one gets the following expression after transformation:

$$
\begin{aligned}
C_{S B E}= & \frac{C_{J E}}{\left(1-F_{C}\right)^{\left(1+M_{J E}\right)}} *\left[1-F_{C} *\left(1+M_{J E}\right)\right] \\
& +\left[\frac{C_{J E}}{\left(1-F_{C}\right)^{\left(1+M_{J E}\right)}} * \frac{M_{J E}}{V_{J E}}\right] * v B E
\end{aligned}
$$

It can be observed that Eq.6 may be considered as a linear function with an independent argument vBE, because other coefficients are constant. Therefore, linear fitting function is constructed as follow:

$$
\begin{gathered}
y=b+a x \\
y=C_{S B E} \\
b=\frac{C_{J E}}{\left(1-F_{C}\right)^{\left(1+M_{J E}\right)}} *\left[1-F_{C} *\left(1+M_{J E}\right)\right] \\
a=\left[\frac{C_{J E}}{\left(1-F_{C}\right)^{\left(1+M_{J E}\right)}} * \frac{M_{J E}}{V_{J E}}\right] \\
x=v B E
\end{gathered}
$$

$F_{C}=0.5$ by default. In order to obtain a parameters value more accurate, an interval $0.5 \mathrm{~V}<\mathrm{vBE}<0.8 \mathrm{~V}$ on the Cje$\mathrm{vBE}$ curve is defined to extract the remaining two parameter values, Vje and Mje. This specific zone is chosen not only for matching the applying condition $\left(\mathrm{vBE} \geq \mathrm{F}_{\mathrm{C}} * \mathrm{~V}_{\mathrm{JE}} \approx 0.5 * 0.6=0.3 \mathrm{~V}\right)$, but also for the reason of the highest linearization degree whose $R^{2}$ value is close to 1 to reduce the additional error. The fitting result is shown in the following figure:

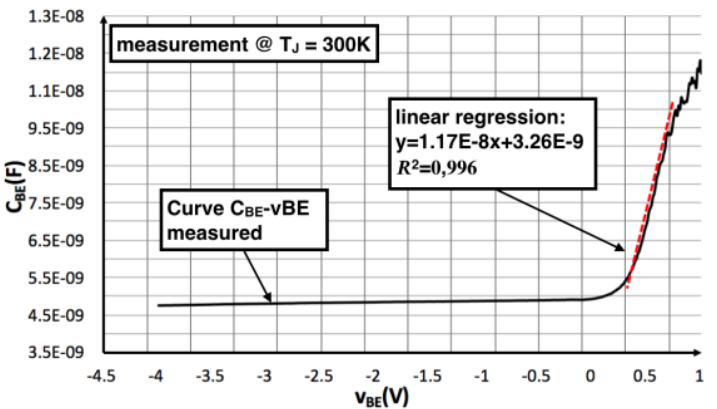

Fig. 6 fitting result on the Cbe-vBE curve between $v B E=0.5 \mathrm{~V}$ and $0.8 \mathrm{~V}$

The results for different chips are given as follow:

Tab-3: extraction results of capacitive parameters

\begin{tabular}{|c|c|c|c|c|}
\hline & Chip- 1 & Chip- & Chip-3 & Average \\
\hline \multirow{2}{*}{ Fitting } & $\mathrm{y}=1.17 \mathrm{E}-8 \mathrm{x}+$ & $\mathrm{y}=1.23 \mathrm{E}-8 \mathrm{x}+$ & $\mathrm{y}=1.35 \mathrm{E}-8 \mathrm{x}+$ & \\
Equation & $3.26 \mathrm{E}-9$ & $3.408 \mathrm{E}-9$ & $3.42 \mathrm{E}-9$ & \\
& $\mathrm{R}^{2}=0.996$ & $\mathrm{R}^{2}=0.996$ & $\mathrm{R}^{2}=0.993$ & \\
\hline $\mathrm{Mje}$ & 0.54 & 0.52 & 0.52 & 0.53 \\
\hline $\mathrm{Cje}(\mathrm{nF})$ & 4.87 & 4.93 & 4.97 & 4.92 \\
\hline $\mathrm{Vje}(\mathrm{V})$ & 0.65 & 0.6 & 0.56 & 0.6 \\
\hline
\end{tabular}

\section{Extraction of the DC parameters}

\section{C.1. Extraction of Var and Vaf}

The Vaf and Var that model transistor's so called 'Early voltage'. They will be the first to be extracted among DC parameters because its dependence with others. The curve $\mathrm{iC}$-vCE is required for the extraction. According to Fig.4, the curve can be obtained by fixing an input iB (here is 10,100 and $100 \mathrm{uA}$ ), varying the voltage vCE and measuring the collector current iC. According to Eq.2 given at the beginning, after some simplifications, one can obtain a new iC expression as follow:

$$
i_{C}=\frac{2 * I s * \exp \left(\frac{v B E}{N F * V T}\right)}{1+\sqrt{1+4 \frac{I S}{I_{K F}} \exp \left(\frac{v B E}{N F * V T}\right)}} * \frac{1}{V_{A F}}\left(V_{A F}+v C E\right)
$$

Referring to this equation, it can be found that parameter Vaf equals to iC-vCE curve's intersection with the $\mathrm{x}$ axis. Based on actual operating experience, different iB values will lead to different Vaf. It is mainly due to the approximation of some effects during simplification process in Eq.8. Therefore, to obtain a more representative Vaf value, numerous base current iB $(10 \mathrm{uA}, 100 \mathrm{uA}, 1 \mathrm{~mA})$ will be selected to compute the Early voltage, the measurement result is $162 \mathrm{~V}$. Reverse 'Early voltage' Var has the same extraction procedure as the forward one. Its measurement result is $5.0 \mathrm{~V}$. The Vaf and Var will be extracted from each measurement to finally compute their average that is considered as their final value and will be applied in the simulations.

\section{C.2. Extraction of Ise(c), $\mathrm{Ne}(\mathrm{c})$ and $\mathrm{Bf}(\mathrm{r})$}

Three parameters play a decisive role on the base current iB. The Ise and Ne impact on low current zone of iB. To the opposite, $\mathrm{Bf}$ influences its linear rising segment, which will indirectly impact on the value of the collector current $\mathrm{iC}$. The extraction of these parameters requires the $\mathrm{iB}-\mathrm{vBE}$ curve and its measuring configuration is the 
same as that of Is, $\mathrm{Nf}$ and $\mathrm{Nr}$ extraction. Firstly, according to the expression iB given in Eq.1, some conditions (vBE around $0.7 \mathrm{~V}$ and $\mathrm{BC}$ junction in reverse bias) are proposed to simplify the computation. Because $\mathrm{BC}$ junction in reverse bias, all exponential terms with $\mathrm{vBC}$ can be ignored. Practical experience shows that the Is and Ise are nA level parameters, which are ignorable before iB. Similarly, in order to improve the accuracy, vBE equals to $0.7 \mathrm{~V}$ indicates that the vBE/Var can't be ignored compared with 1 . In this case, the final base current iB can be written as:

$$
i_{B}=\frac{1}{B F} * \frac{i_{C}}{\left(1-\frac{v B E}{V_{A R}}\right)}+I s e * \exp \left[\frac{v B E}{N E * v t}\right]
$$

The extraction of these parameters needs to use the recursive method. Before applying this method, one additional term $E_{r e l}$ will be added into Eq.9 representing the error between theoretical value and measurement.

$$
E_{\text {rel }}=\frac{1}{B F * i_{B i}} * \frac{i_{C_{i}}}{\left(1-\frac{v B E_{i}}{V_{A R}}\right)}+\frac{I s e}{i_{B_{i}}} * \exp \left[\frac{v B E_{i}}{N E * v t}\right]-1
$$

With Eq.10, a new specific function called 'cost function' is proposed as below. 'cost function' is a function that expresses the error between model theory value and actual measurement data. If the 'cost function' gets 0 , it means that the model matches perfectly with the measurement.

$E_{\text {tot }}=\sum_{i=1}^{N}\left[\frac{1}{B F * i_{B i}} * \frac{i_{C_{i}}}{\left(1-\frac{v B E_{i}}{V_{A R}}\right)}+\frac{I s e}{i_{B_{i}}} * \exp \left[\frac{v B E_{i}}{N E * v t}\right]-1\right]^{2}$

Assuming that:

$$
i_{C_{i}}^{\prime}=\frac{i_{C_{i}}}{\left(1-\frac{v B E_{i}}{V_{A R}}\right)}
$$

One calculates the partial derivation of Eq.11 versus Bf and Ise. With the getting derivation results, after simplifications, the Ise can be deduced as follow:

$$
=\frac{\sum_{i=1}^{N} \frac{1}{i_{B_{i}}} \exp \left[\frac{v B E_{i}}{N E * v t}\right] * \sum_{i=1}^{N} \frac{i_{C_{i}}^{\prime 2}}{i_{B_{i}}{ }^{2}}-\sum_{i=1}^{N} \frac{i_{C_{i}}^{\prime}}{i_{B_{i}}} * \sum_{i=1}^{N} \frac{i_{C_{i}}^{\prime}}{i_{B_{i}}{ }^{2}} \exp \left[\frac{v B E_{i}}{N E * v t}\right]}{\sum_{i=1}^{N} \frac{1}{i_{B_{i}}{ }^{2}} \exp \left[\frac{2 v B E_{i}}{N E * v t}\right] * \sum_{i=1}^{N} \frac{i_{C_{i}}^{\prime 2}}{i_{B_{i}}{ }^{2}}-\left[\sum_{i=1}^{N} \frac{i_{C_{i}}^{\prime}}{i_{B_{i}}{ }^{2}} \exp \left[\frac{v B E_{i}}{N E * v t}\right]\right]^{2}}
$$

Similarly, the Bf can be separated to obtain its expression as:

$$
B F=\frac{\sum_{i=1}^{N} \frac{i_{C_{i}}^{\prime 2}}{i_{B_{i}}{ }^{2}}}{\sum_{i=1}^{N} \frac{i_{C_{i}}^{\prime}}{i_{B_{i}}}-I s e * \sum_{i=1}^{N} \frac{i_{C_{i}}^{\prime}}{i_{B i}{ }^{2}} \exp \left[\frac{v B E_{i}}{N E * v t}\right]}
$$

Now it can be observed that only Ne remains unknown in Eq.11. Therefore, a starting value of the $\mathrm{Ne}$ will be selected and will be substituted into the $\mathrm{Bf}$ and Ise expression. Then, the three parameters ( $\mathrm{Bf}$, Ise and $\mathrm{Ne}$ ) will be substituted together into Eq.11 to compute the value of 'cost function'. One Repeats the above steps and constantly changes the value of Ne until making the 'cost function' the minimum, which means that the theoretical model is the closest to the actual measurements. The average value is calculated as the final model parameter:
Tab-6: extraction results of $\mathrm{Ne}$, Ise and $\mathrm{Bf}$

\begin{tabular}{|c|c|c|c|c|}
\hline & Chip-1 & Chip-2 & Chip-3 & Average \\
\hline $\mathrm{Ne}$ & 1.967 & 1.973 & 1.972 & 1.971 \\
\hline Ise(A) & $1.29 \mathrm{E}-9$ & $1.33 \mathrm{E}-9$ & $1.30 \mathrm{E}-9$ & $1.30 \mathrm{E}-9$ \\
\hline Bf & 325 & 326 & 323 & 325 \\
\hline
\end{tabular}

The reverse parameters extraction method is totally the same as that of forward parameters, just be attention at using the curve iB-vBC instead of the iB-vBE. After computation, the extraction result is as follow:

Tab-7: extraction results of $\mathrm{Nc}$, Isc and $\mathrm{Br}$

\begin{tabular}{|c|c|c|}
\hline $\mathrm{Nc}$ & $\mathrm{Isc}(\mathrm{A})$ & $\mathrm{Br}$ \\
\hline 1.48 & $1.36 \mathrm{E}-9$ & 45 \\
\hline
\end{tabular}

\section{C.3. Extraction of Ikf and Ikr}

These parameters present the current roll-off phenomenon when it is high. The extraction of Ikf will use all the parameters that have been extracted previously. So, its extraction process must be very careful to ensure its value in a reasonable range as well as to ensure the success of simulation. The extraction of Ikf needs two groups of measured data: $\mathrm{iC}-\mathrm{vBE}$ and $\mathrm{iB}-\mathrm{vBE}$; the measuring configuration is the same as that used in Is, $\mathrm{Nf}$ and $\mathrm{Nr}$ extraction.

To simplify the extraction, some conditions (vBE around $0.7 \mathrm{v}$ and $\mathrm{BC}$ junction in reverse bias) should be respected. The amplification expression of a transistor $h_{F E}$ equals to the ratio of $\mathrm{iC}$ and $\mathrm{iB}$, which are replaced by their respective mathematic equations to obtain a new amplification expression:

$$
h_{F E}=\frac{i_{C}}{i_{B}}=\frac{1-\frac{v B E}{V_{A R}}-\frac{v B C}{V_{A F}}-\frac{i_{C}}{I_{K F}}}{\frac{1}{B F}+\frac{I s e}{I s} * \exp \left[\frac{v B E}{v t}\left(\frac{1}{N E}-\frac{1}{N F}\right)\right]}
$$

In Eq.15, only parameter Ikf is unknown. The same method used in the extraction of $\mathrm{Ne}$, Ise and $\mathrm{Bf}$ will be employed again here to identify the value of Ikf. According to the expression of $h_{F E}$, a new 'cost function' is constructed:

$$
E_{\text {tot }}=\sum_{i=1}^{N}\left[\frac{i_{B_{i}}}{i_{C_{i}}} * \frac{1-\frac{v B E_{i}}{V_{A R}}-\frac{v B C_{i}}{V_{A F}}-\frac{i_{C_{i}}}{I_{K F}}}{\frac{1}{B F}+\frac{I S e}{I S} * \exp \left[\frac{v B E_{i}}{v t}\left(\frac{1}{N E}-\frac{1}{N F}\right)\right]}-1\right]^{2}
$$

The extraction principle is to continuously iterate the new Ikf and to calculate the value of 'cost function' after each iteration until getting its minimum. At this moment, the Ikf gets its optimal value and it also means that the model parameters best meet the actual measurements. The parameter Ikf begins to influence the collector current iC when its value exceeds a certain intensity. Based on this principle and aimed to make the extraction more reasonable, this article selects the data segment of $\mathrm{vBE} \geq 0.7 \mathrm{~V}$ to ensure the collector current iC working under high intensity.

As shown in Fig.7, after certain iterations, the 'cost function' gets its minimum when the Ikf equals to $13 \mathrm{~A}$. 
The same extraction method is applied in Ikr extraction. According to computation, when Ikr equals to $1.75 \mathrm{~A}$, the 'cost function' gets the minimum.

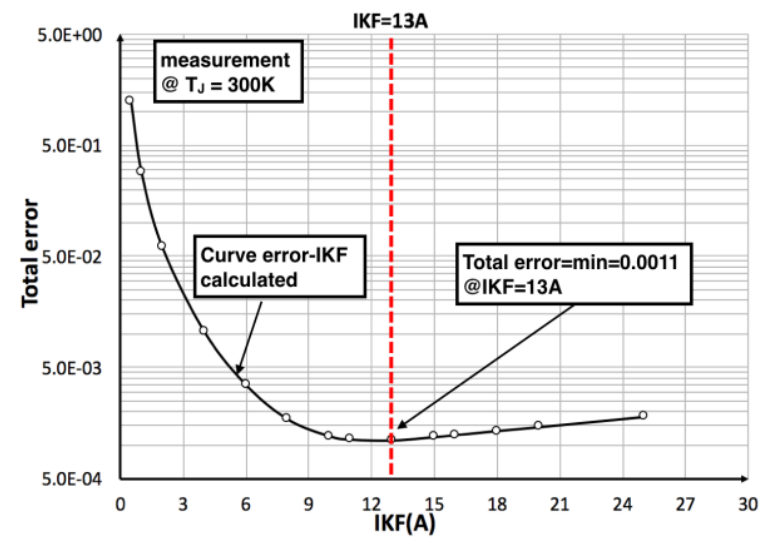

Fig.7 finding the optimal IKF value by doing iteration to make Total error become the minimum

\section{The simulation and verification of the model}

In the third part, the parameter extraction methods of Gummel-poon model have been introduced one after another. After obtaining all the parameters, in this part, the validation of the model will be performed by comparing the simulation results with experimental ones. The simulation in Pspice will be divided into two parts: simulation of a single Super-Gain BJT and simulation of the TBBS. The simulation results are as follows:

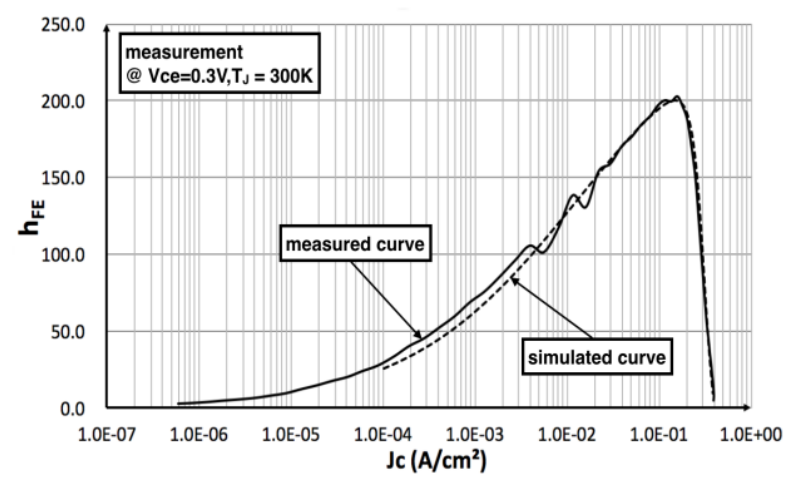

Fig.8 comparison of simulated Gummel curve of a single supergain BJT with measured one in forward bias

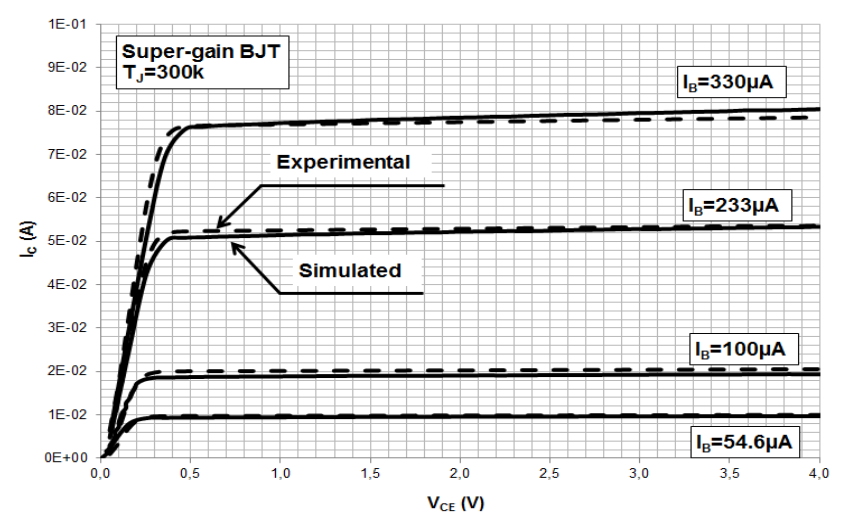

Fig.9 comparison of simulated output curve of a single supergain BJT with measured one in forward bias

We can observe, for the Gummel curve and output curve of the Super-Gain BJT, that the 'Absolute Total Error' between the simulated and the measured curve is less than $10 \%$. It proves that the model for the single SuperGain BJT proposed in this article corresponds completely to its actual electronic characteristics.

\section{A new TBBS model and its simulations}

In order to establish the TBBS electrical model by using the signal super-gain BJT model, a new Pspice simulation model is proposed. In the new model, two additional resistances Rcp and Rep are connected respectively in parallel with the collector terminal $\mathrm{C}$ and the emitter terminal $\mathrm{E}$ as shown in Fig. 10.

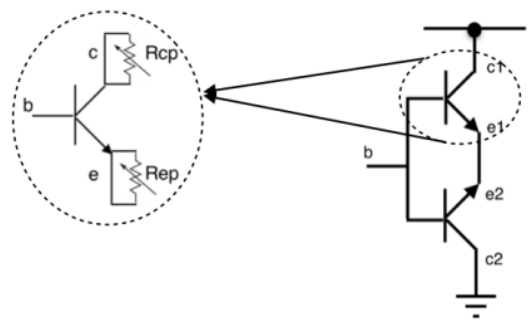

Fig.10 a new TBBS model with two additional resistances

From this paragraph, some simulations based on the new TBBS model will be performed. Before launching the simulations, values of the resistors Rcp and Rep should be calculated. By constantly varying their resistances, at each variation, its 'Absolute Total Error' of the Gummel curve should be calculated to find the optimum resistances values that makes the $\boldsymbol{E}_{\boldsymbol{a b s}}(\boldsymbol{t o t})$ minimum. Calculating result is as below:

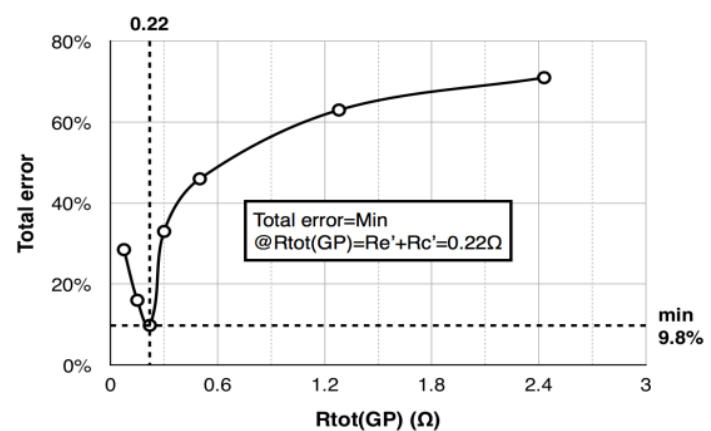

Fig.11 total error curve versus Rtot(GP) to find the optimum resistance value making the total error become minimum

According to the calculation, when the $\mathrm{R}_{\text {tot }}(\mathrm{GP})$ takes $0.22 \Omega$, the Total Error becomes the minimum. In this case, $\mathrm{Rc}=0.15 \Omega, \mathrm{Re}^{\prime}=0.07 \Omega$, and the parallel resistors $\mathrm{Rcp}=0.262 \Omega$, Rep $=0.108 \Omega$. Using the new resistances $\mathrm{Rc}=0.15$ and $\mathrm{Re}=0.07 \Omega$, the simulation result of TBBS in Pspice is presenting in Fig. 12:

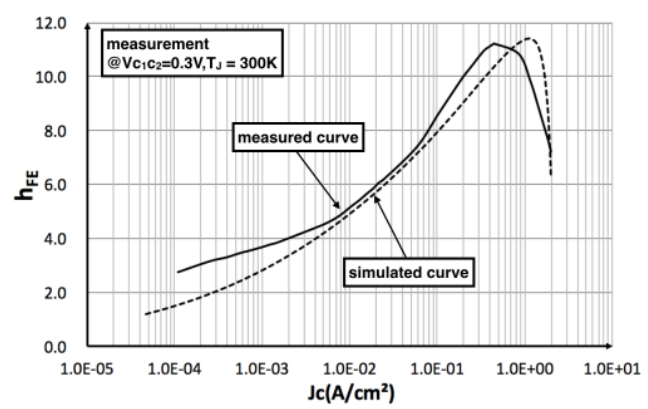

Fig.12 comparison of the simulated Gummel curve with the 
measured one (new TBBS model)

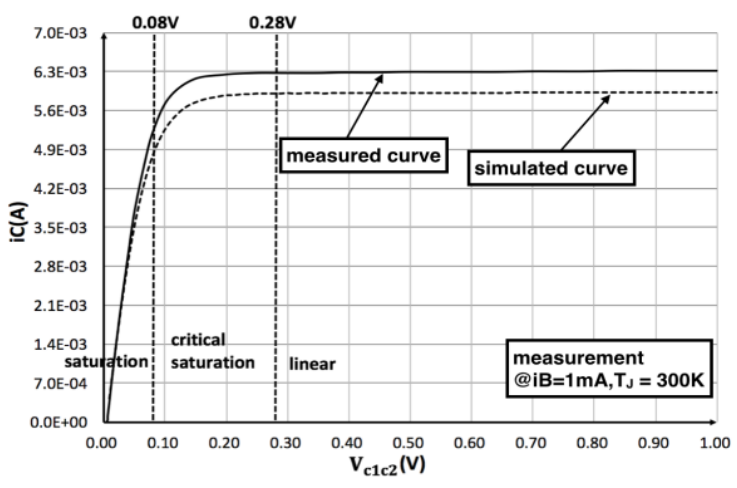

Fig. 13 comparison of the simulated output curve with the measured one (new TBBS model)

The absolute total error for the Gummel curve and the output curve are respectively $9.8 \%$ and $7.9 \%$, it demonstrates that the new model fits well to the component's electronic characteristics. Thus, its validation is confirmed.

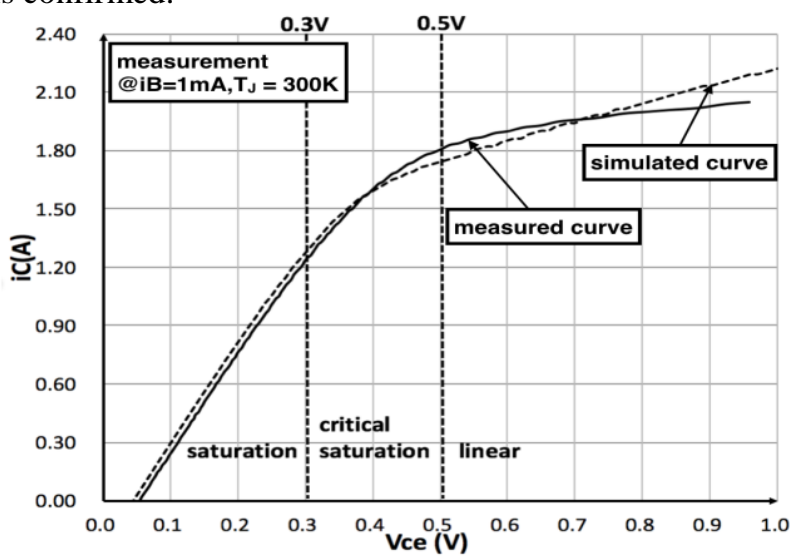

Fig. 14 output curve simulation of TBBS in $i B=500 \mathrm{~mA}$ with new model

As a new power switch, its collector current iC will reach in a magnitude of $500 \mathrm{~mA}$. Therefore, a new TBBS simulation will be executed in $\mathrm{iB}=500 \mathrm{~mA}$ to verify its performance in high current and the result is as follow:

The Absolute Total Error calculated from the simulation is $3.2 \%$ and the simulation parameters are as follows:

$\mathrm{Br}=45 \mathrm{Ikr}=1.75 \mathrm{~A} \mathrm{Nc}=1.48, \mathrm{Nr}=1.05$, Isc=1.36n, Var=5, $\mathrm{Is}=0.13 \mathrm{n}$ Ise $=1.3 \mathrm{n} \mathrm{Ne}=1.95 \mathrm{Nf}=1.053 \mathrm{Bf}=325 \mathrm{Vaf}=162$ $\mathrm{Mje}=0.53 \mathrm{Cje}=4.92 \mathrm{E}-9 \mathrm{Vje}=0.60 \mathrm{Mjc}=0.32 \mathrm{Cjc}=0.92 \mathrm{E}-9$ $\mathrm{Vjc}=0.53 \mathrm{Ikf}=13 \mathrm{Re}=0.035 \mathrm{Rc}=0.04 \mathrm{Rb}=3.022$

\section{Conclusion and prospective}

On the basis of the research of the new Super-Gain BJT developed by laboratory GREMAN, this article introduces in a detailed method for modeling the Super-Gain BJT and of extracting its model parameters. Simultaneously, the single BJT and the power switch TBBS (two Super-Gain BJTs connected in anti-series) are simulated in Pspice to validate their electrical models. According to the simulation results, a new TBBS simulation model and its interpretation is given to overcome the defect of GummelPoon model. Consequently, in all simulation cases, the
'Absolute Total Error' is less than $10 \%$, it fully proves that the model used in this article and its extracted parameters are correct.

It is a great challenge to simulate the Super-Gain BJT with Gummel-Poon model in hi-level injection. The simulation result from Pspice indicates that the proposed model becomes more erroneous when current density Jc is higher than $2.0\left(\mathrm{~A} / \mathrm{cm}^{2}\right)$ principally due to the influence of resistances. To extend the validation scope to a lager one, furthermore, researchers should focus on modeling the high-current injection part of Super-Gain BJT. Against the shortcoming of Gummel-Poon model, we propose an efficient modified method to improve its performance in modeling the Super-Gain BJT. That is replacing the fixed-value resistance by a current-depend resistance in the model for the aim of simulating the resistance effect and of making up for deficiencies of Gummel-Poon model. This resistance will change its value according to the current flowing through it and definitively make the simulations more flexible and more accurate. The simulation results of this new model have proved its robustness.

\section{REFERENCES}

[1] C. Benboujema, S. Jacques, A. Schellmanns, N. Batut, J.-B. Quoirin, L. Jaouen, L. Ventura, Characterization of a high gain BJT used in power conversion on AC mains, Proceedings of the IEEE Energy Conversion Congress and Exposition, 2010, pp. 357-361.

[2] Rectifier and controller including triac switch. U.S. Patent No 3,421,063, 7 janv. 1969.

[3] Hefner, A. R. (1990, June). An investigation of the drive circuit requirements for the power insulated gate bipolar transistor (IGBT). In Power Electronics Specialists Conference, 1990. PESC'90 Record., 21st Annual IEEE (pp. 126-137). IEEE.

[4] Lundstrom, M. (1997). Elementary scattering theory of the Si MOSFET. IEEE Electron Device Letters, 18(7), 361-363.

[5] Holtz, J., Lammert, P., \& Lotzkat, W. (1987). High-speed drive system with ultrasonic MOSFET PWM inverter and single-chip microprocessor control. IEEE transactions on industry applications, (6), 1010-1015.

[6] REN Zheng, SCHELLMANNS Ambroise, BATUT Nathalie, "Development and Static Mode Characterization of a New Lowloss AC Switch Based on Super-Gain BJT", Special issue published in Journal of Energy and Power Engineering in January 2014.

[7] L.-V. Phung, Chawki Benboujema, Jean-Baptise Quoirin, BATUT Nathalie, Modeling of a new SOI bidirectional bipolar junction transistor for low-loss household appliances, IEEE Transactions on Electron Devices, Vol. 58, No 4, 2011, pp. 1164-1169.

[8] REN Zheng, SCHELLMANNS Ambroise, BATUT Nathalie. Dynamic Mode Characterization of a New Super-Gain BJT and an Innovative Low-Loss AC Switch. In: 2016 IEEE Workshop on Microelectronics and Electron Devices (WMED). IEEE, 2016. p. $1-4$.

[9] Franz Sischka, "Gummel-Poon Bipolar Model, model description and parameter extraction", Agilent Technologies GmbH, 2006 , Munich (Germany) 